BMJ Open Sport \& Exercise Medicine

\title{
Patient pain drawing is a valuable instrument in assessing the causes of exercise-induced leg pain
}

Kajsa Rennerfelt, Qiuxia Zhang, Jón Karlsson, Jorma Styf

To cite: Rennerfelt $\mathrm{K}$, Zhang $\mathrm{Q}$, Karlsson J, et al. Patient pain drawing is a valuable instrument in assessing the causes of exerciseinduced leg pain. BMJ Open Sport \& Exercise Medicine 2018;4:e000262. doi:10.1136/ bmjsem-2017-000262

Accepted 17 December 2017

\section{ABSTRACT}

Aim We validated patientpain drawing (PPD) in establishing the diagnosis of chronic anterior compartment syndrome (CACS) in patients with exercise-induced leg pain.

Methods The study comprised 477 consecutive patients, all suspected of having CACS. The diagnosis was based on the patient's history, a thorough clinical examination and measurements of intramuscular pressure (IMP) following an exercise test. Patients completed a PPD before their hospital visit. Two independent orthopaedic surgeons diagnosed the causes of leg pain based only on the PPD at least 1 year after admission. Based on the results of diagnostic tests, the patients were divided into three groups: CACS $(n=79)$, CACS with comorbidity $(n=89)$ and non-CACS $(n=306)$.

Results The sensitivity of the PPD to identify CACS correctly was $67 \%$ (observer 1) and $75 \%$ (observer 2). The specificity was $65 \%$ and $54 \%$, respectively. The interobserver agreement $(n=477)$ was $80 \%$, and the kappa value was 0.55 . The interobserver agreement was $77 \%$, and the kappa value was 0.48 among 168 CACS patients with or without comorbidity. The interobserver agreement was $85 \%$, and the kappa value was 0.56 in 79 CACS, and CACS was correctly diagnosed in $79 \%$ (observer 1) and $82 \%$ (observer 2). The test-retest showed the same results for the two observers, with an intraobserver agreement of $84 \%$, while the test-retest reliability coefficient was 0.7 . Comorbidity was found in 53\% of CACS patients.

Conclusion PPD might be a valuable instrument in diagnosing the causes of exercise-induced leg pain. It is useful in identifying CACS with and without comorbidity.

\section{INTRODUCTION}

Palmer introduced the concept of patient Department of Orthopaedics, Institute of Clinical Sciences, Sahlgrenska Academy, Sahlgrenska University Hospital, University of Gothenburg, Gothenburg, Sweden

\section{Correspondence to} Dr Kajsa Rennerfelt, Department of Orthopaedics, Institute of Clinical Sciences, Sahlgrenska Academy Sahlgrenska University Hospital, University of Gothenburg Gothenburg Sweden ; kajsa.rennerfelt@vgregion.se pain drawing (PPD) in 1949. The basic idea was to differentiate between functional and organic pain by using a pain chart made by the patient as a diagnostic instrument. ${ }^{1}$ PPD has been used first and foremost in the diagnostic evaluation of patients with low back pain, ${ }^{2}$ and it has shown high interobserver reliability and intra-observer agreement. However, the clinical usefulness of PPD in diagnosing the causes of exercise-induced leg pain and its sensitivity and specificity as a diagnostic tool has not been reported before.

\section{What are the new findings?}

- In patients with isolated chronic anterior compartment syndrome, patient pain drawing (PPD) shows an intraobserver agreement of more than $80 \%$ among the observers in correct assessment.

- More than $50 \%$ of patients with chronic compartment syndrome have one or more additional diagnosis.

- Patients with chronic anterior compartment and comorbidity might improve the outcome of surgery if PPD is routinely used.

Exercise-induced leg pain is a common problem in athletes, ${ }^{3-5}$ and it results from various conditions that involve different tissues, such as bones, muscles, blood vessels, nerves and tendons. Comorbidity has also been reported in many patients with exercise-induced leg pain. ${ }^{6}{ }^{7}$ In a retrospective review of 150 athletes with exercise-induced leg pain, chronic compartment syndrome was the most common condition with a prevalence of $33 \%$. In the same report, $25 \%$ of the athletes had stress fractures, $13 \%$ had medial tibial stress syndrome and $10 \%$ had nerve entrapment syndromes. ${ }^{8}$ These conditions can often coexist, making an accurate diagnosis difficult. To establish an accurate diagnosis, therefore, requires obtaining a thorough patient history, performing a comprehensive physical examination, reproduction of symptoms with exercise and coordinating the appropriate diagnostic studies to further distinguish between the different conditions. ${ }^{9}$

Chronic compartment syndrome is an underdiagnosed cause of chronic exertional leg pain, and the syndrome is induced by exercise that impairs neuromuscular function within the involved compartment. ${ }^{10}$ The anterior compartment of the leg is the most commonly involved, followed by the lateral compartment, the deep posterior compartment and finally the superficial posterior compartment. ${ }^{11}$ Chronic anterior compartment syndrome (CACS) is characterised by 
the onset of leg pain after a specific amount of exercise and occurs only in the anterior aspect of the leg. ${ }^{12}$ The idea of using PPD as a screening instrument in patients with exercise-induced leg pain may help to assess the location of pain and distinguish anterior pain from posterior pain, in order to screen patients for pressure recording and to overview the comorbidity for diagnosing patients with overuse injuries of the leg. As the patients are symptom free at rest, a screening test would also be useful in general orthopaedic practice.

The purpose of this study was to validate PPD as an additional instrument for identifying CACS in patients with exercise-induced leg pain. The hypothesis is that PPD is a valuable diagnostic instrument for identifying CACS.

\section{METHODS \\ Patients}

The study comprised 477 consecutive patients, 258 men and 219 women, with a median age of 31 (range 15-70) years, all with exercise-induced leg pain and clinical signs of possible CACS. Most of the patients were involved in running activity several times a week or some other type of high-level organised sports. All patients were referred to the orthopaedic department between May 2009 and October 2013 for an evaluation of their leg pain. The patients were divided into different groups (CACS, CACS with comorbidity and non-CACS), according to diagnoses, for analytical purposes (figure 1).

\section{Observers}

Two independent orthopaedic surgeons (observers 1 and 2) established the diagnoses of the causes of leg pain based only on the PPD drawing. To avoid recall bias, the procedure was performed at least 1 year after the patient's clinical appointment. In relation to the drawings, the observer was able to select one or more diagnoses from a list of six diagnoses and one group of miscellaneous, that is, CA, chronic lateral compartment syndrome, chronic posterior compartment syndrome, medial tibial syndrome (MTS), peroneal tunnel syndrome (PTS), muscle rupture and a group of miscellaneous.

\section{Patient pain drawing}

All the patients completed a questionnaire, which they received by mail 2 weeks prior to their visit to the orthopaedic department. The questionnaire included an outline of a human body with anterior, posterior and lateral views (figure 2). The patients indicated the afflicted areas of pain on the drawing and either single locations or larger regions could be marked. The PPD was later discussed during the meeting with the doctor.

The assessments of the drawings were divided according to the different diagnoses mentioned above. A drawing indicating pain on the anterior aspect of the leg was defined as chronic anterior compartment syndrome. Laterally, a general indicated area was assessed as lateral chronic compartment syndrome ${ }^{67}$ while a local

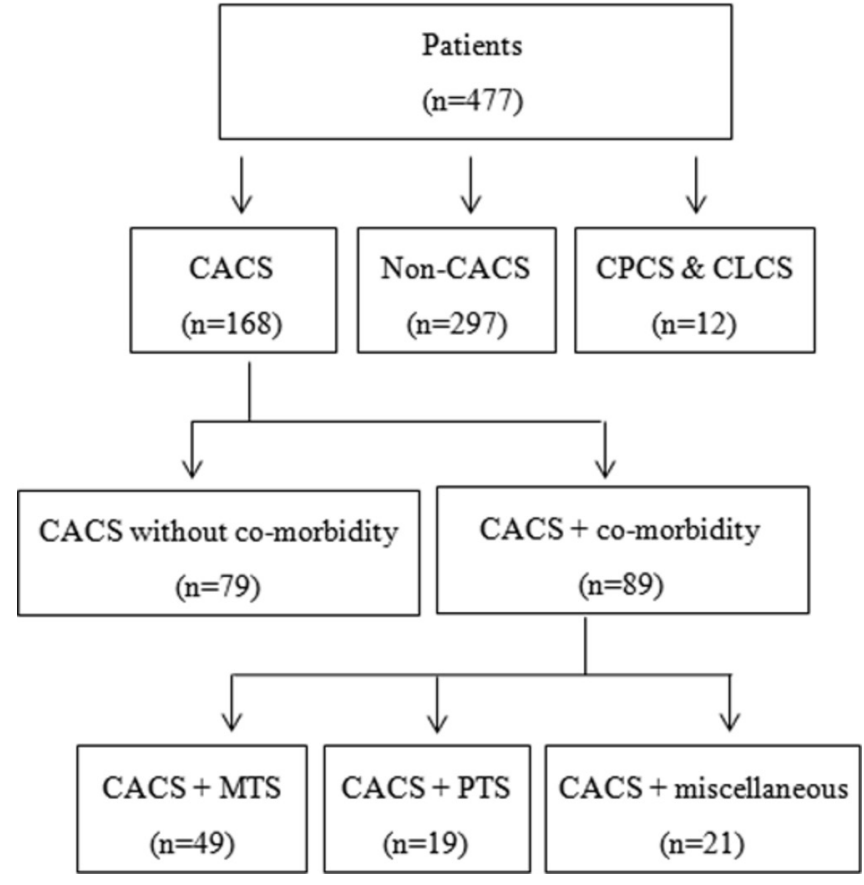

Figure 1 The 477 patients were allocated to the following groups: (1) CACS; (2) CACS with comorbidity; and (3) nonCACS, patients with other causes of exercise-induced leg pain. 'Miscellaneous' includes muscle strains and partial muscle rupture, popliteal entrapment, venous insufficiency and suspected stress fractures. CACS, chronic anterior compartment syndrome; CLCS, chronic lateral compartment syndrome; CPCS, chronic posterior compartment syndrome; MTS, medial tibial syndrome; PTS, peroneal tunnel syndrome.

indication in the distal third of the lateral aspect of the leg was assessed as PTS. ${ }^{13}$ MTS was assessed as a pain drawing indicated medially over the distal third of the $\operatorname{leg}^{14}{ }^{15}$ and, in some cases, over the dorsal aspect of the foot. On the posterior aspect of the leg, muscle rupture was assessed as a local indication over the proximal portion of the leg, while posterior compartment syndrome was assessed as a more general posterior indication on the pain drawing. Before the assessments by the observers were made, a pretest comprising 10 drawings was conducted to discuss the way the drawings should be interpreted.

One year after the primary assessment, the same observers re-evaluated 100 PPD forms to estimate the test-retest reliability.

\section{Diagnostic criteria}

\section{Chronic anterior compartment syndrome}

The correct diagnosis of CACS was regarded as the one registered by the doctor in the medical records, which was based on the patient's history, a complete clinical examination and measurements of intramuscular pressure (IMP) following an exercise test that elicited the patient's symptoms. The diagnostic criteria for CACS included the following: (1) exercise-induced leg pain with reversed symptoms at rest, (2) impaired muscle function during activity, (3) swelling and tenderness over 

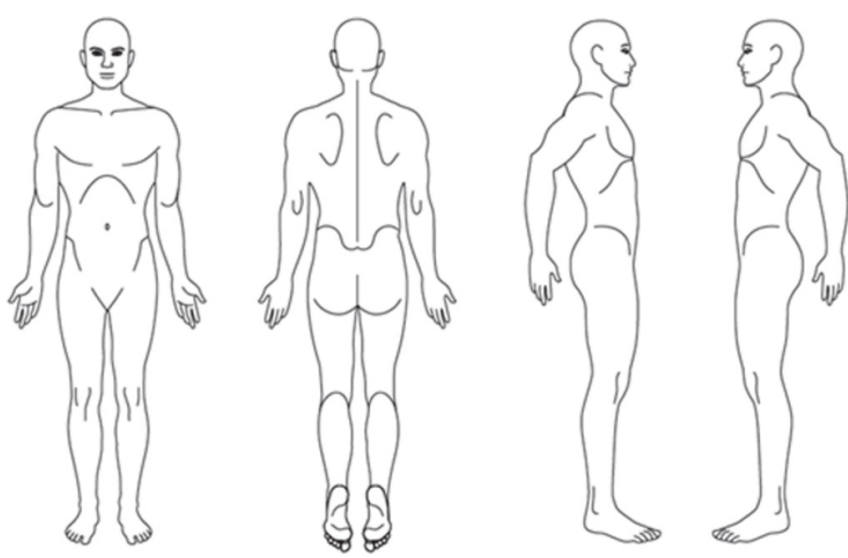

Figure 2 Anteroposterior and lateral views of a human outline used for patient pain drawing.

the anterior compartment immediately after exercise, (4) IMP of $\geq 30 \mathrm{~mm} \mathrm{Hg} 1 \mathrm{~min}$ after exercise and (5) IMP of $\geq 20 \mathrm{~mm} \mathrm{Hg} 5$ min after exercise. ${ }^{16}$

\section{Medial tibial syndrome}

It was defined as exercise-induced pain along the posteromedial border of tibia, soreness at palpation over five or more consecutive centimetres. ${ }^{17}$

\section{Peroneal tunnel syndrome}

A PTS was diagnosed when there were decreased sensibility and pain over the dorsum of the foot at rest or during exercise. Pain at palpation, where the nerve emerges through the fascia, also confirmed the diagnosis.

Miscellaneous includes muscle strains and partial muscle rupture, popliteal entrapment, venous insufficiency and suspected stress fractures; this group of diagnoses appeared with pain in the calf that increases during activity. Ultrasound-verified muscular damage or oedema indicated inflammatory reaction. Venous insufficiency such as varicose veins or other insufficiency diagnosed by ultrasound. Popliteal entrapment diagnosed by MRI. Stress fractures were diagnosed by radiological findings using radiographs or MRI.

\section{Exercise test}

All the patients performed an exercise test after the clinical examination. The test was individualised and aimed to elicit and reproduce the patient's leg pain and symptoms. The test started with running up and down stairs or running outside in the park or on a treadmill, followed by repeated concentric dorsiflexion of the ankle joints in a standing position until the patient was unable to continue due to muscle exhaustion and/or leg pain.

\section{Monitoring IMP}

IMP was measured within $60 \mathrm{~s}$ after the exercise test in all patients. IMP was measured with the patient in the supine position using a microcapillary infusion system (Hemo 4, Siemens, Erlangen, Germany) and monitor (Siemens SC 9000, Siemens, Gothenburg, Sweden). ${ }^{18}$ The skin was penetrated with a $1.2 \mathrm{~mm}$ diameter needle
Table 1 Sensitivity and specificity of PPD to identify CACS in patients with exercise-induced leg pain $(n=477)$

\begin{tabular}{lll}
\hline Variable & Observer 1 & Observer 2 \\
\hline Sensitivity (\%) & 75 & 67 \\
Specificity (\%) & 54 & 65 \\
Positive predictive value (\%) & 47 & 51 \\
Negative predictive value (\%) & 80 & 78
\end{tabular}

CACS, chronic anterior compartment syndrome; PPD, patient pain drawing.

with four side holes at its tip. The needle was inserted at a $30^{\circ}$ angle to the long axis of the leg in the distal direction to the belly of the anterior tibial muscle ${ }^{19}$ in the most symptomatic leg in all patients. A non-infusion of $0.9 \%$ saline solution was performed to maintain the bulging of fluid at the tip of the needle. The tip of the catheter and the transducer were placed at heart level to minimise the hydrostatic artefacts, and the position of the tip of the catheter was controlled by ultrasound. The patient lay supine in a relaxed position with his/her legs straight. ${ }^{20}$

\section{Statistical analysis}

Student's t-test was used to determine the significance of intergroup differences. A $\mathrm{P}$ value of $\leq 0.05$ defined significance. The sensitivity and specificity of PPD was calculated in relation to the correct diagnosis based on the complete clinical examination. The level of agreement between the observers was analysed using Cohen's kappa test. The kappa test is a measurement of agreement for variables that can be categorised. Interobserver agreement was assessed.

\section{RESULTS}

IMP was 48 (SD 16) $\mathrm{mm} \mathrm{Hg}$ in the 168 patients with CACS, while it was 17 (SD 6) $\mathrm{mm} \mathrm{Hg}$ in the 256 patients without CACS.

The sensitivity, specificity and positive and negative predictive values based on PPD in diagnosing CACS among the 477 patients are shown in table 1 .

\section{Intraobserver agreement}

When assessing the agreement between the PPD and the clinical diagnose that was based on IMP and clinical findings, the correct diagnoses were established in $79 \%$ (observer 1) and $82 \%$ (observer 2) of the patients with CACS $(\mathrm{n}=79)$. The agreement was $80 \%$ (observer 1 ) and $75 \%$ (observer 2 ) in the CACS patients with comorbidity $(\mathrm{n}=89)$.

\section{Interobserver reliability}

Kappa values for the two observers and percentage of agreement between the two observers are shown in table 2.

\section{Intraobserver agreement (test-retest)}

One hundred PPD forms were re-evaluated in a testretest. The intraobserver agreement was $84 \%$ for both 


\begin{tabular}{|c|c|c|}
\hline Variable & Agreement (\%) & Kappa \\
\hline All patients $(n=477)$ & 80 & 0.55 \\
\hline CACS $(n=168)$ & 77 & 0.48 \\
\hline CACS without comorbidity $(n=79)$ & 85 & 0.56 \\
\hline CACS with comorbidity $(n=89)$ & 79 & 0.34 \\
\hline
\end{tabular}

CACS, chronic anterior compartment syndrome.

observers, and the test-retest reliability coefficient was 0.7 .

\section{Comorbidity}

In the present study, $53 \%$ of the patients with CACS were diagnosed with comorbidity. The distribution of the comorbidity among the patients with CACS $(\mathrm{n}=89)$ was MTS in 55\%, PTS in 21\% and miscellaneous in $24 \%$.

\section{DISCUSSION}

The present study shows that the intraobserver agreement in correctly assessed PPD compared with the gold standard varied between $79 \%$ and $82 \%$ in identifying CACS and $75 \%-80 \%$ in identifying CACS with comorbidity. The fact that PPD can be used as an additional tool in assessing the diagnosis of CACS makes it valuable in the clinical assessment of patient with exercised-induced leg pain.

\section{Sensitivity}

The sensitivity ranged between $67 \%$ and $75 \%$ between the two observers. This was somewhat lower than expected and could be explained by several factors. A correct diagnosis of CACS was made by the two observers in about $70 \%$ of patients. The reason for this might be that the patient becomes confused during the process of filling out the drawing and uses the posterior side instead of the anterior when indicating a pain location in the leg on the PPD form. The anterolateral area may be difficult for patients to indicate the pain locations on the human outline. The patient's experience of pain is not easy to draw, and it could also be an opportunity for the patient to make an excessive drawing in order not to miss any important pain locations or to underline severe pain even if the painful area is limited in reality. To increase the sensitivity and to obtain a more organised drawing from the patient, the human outline might need to be more specific and allow a more detailed pain drawing on the leg. The human outline used in this study might be insufficient for the patient's need to illustrate leg pain. A grid might be helpful for the patients to identify specific painful areas, and also a more detailed guidance in text may enhance the quality of the pain drawing. Clinical knowledge of the patient has been shown to influence the kappa value and the interobserver reliability. ${ }^{21}$ The test-retest was therefore performed at least 1 year after the patient visited the clinic in order to avoid recall bias.

\section{Interobserver reliability}

The results show that the interobserver agreement of PPD varied between the different patient groups, CACS without comorbidity and CACS with comorbidity. The kappa values are approximately 0.5 , which is interpreted as moderate. The agreement between the two observers assessing the different groups ranged between $77 \%$ and $85 \%$. The highest agreement between the observers $(85 \%)$ was found when assessing CACS patients without comorbidity. Most patients marked a single pain location on the anterior view of the leg (figure 3). The interobserver agreement was acceptable and shows that the highest values were found in patients without comorbidity. After 1 year, the agreement was $84 \%$ for both observers, and the test-retest reliability coefficient was 0.7 . The results indicate that PPD is a reliable instrument over time and that time does not influence the results to any great extent. It also shows that the two observers have the same intrarater agreement.

\section{Comorbidity}

PPD is a useful instrument that clearly distinguishes between anterior and posterior pain and further analysis revealed that it is valuable in providing guidance in the clinical diagnostic evaluation. PPD identifies comorbidity and is also useful to determine which muscular compartment should be measured by IMP.

Exercise-induced leg pain is difficult to diagnose due to several factors. One factor might be the high incidence
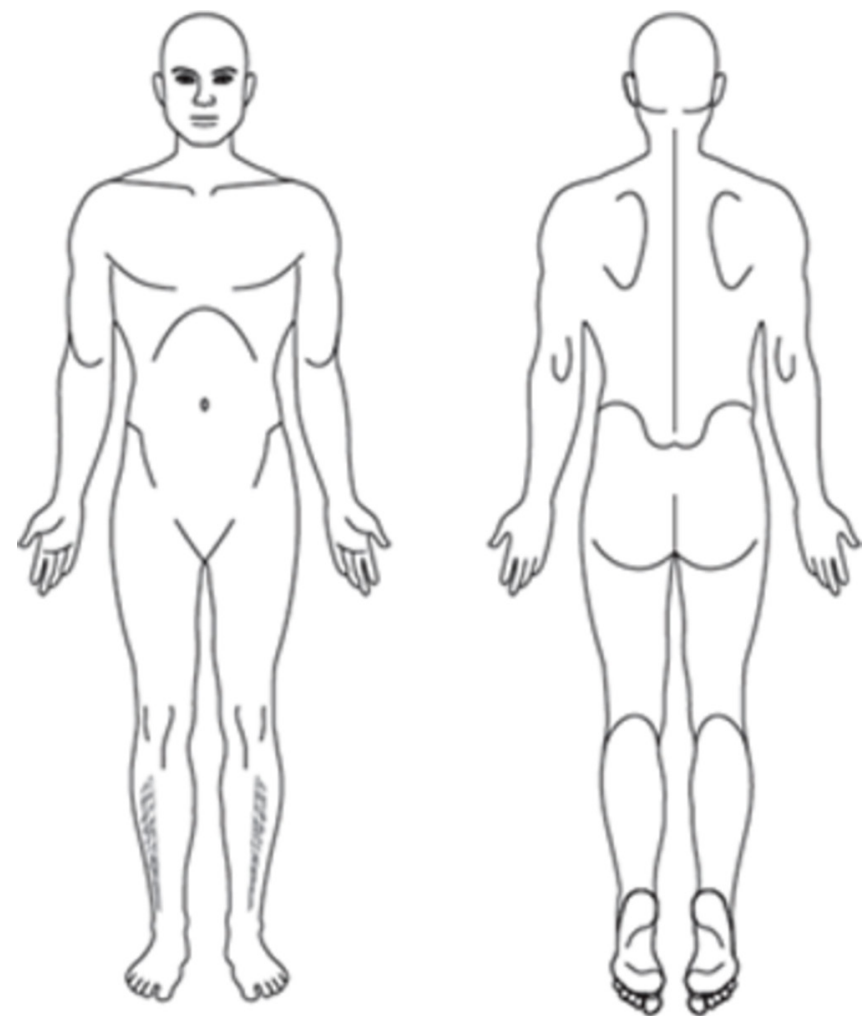

Figure 3 An illustration of a patient pain drawing on a human outline made by a patient with chronic anterior compartment syndrome. 

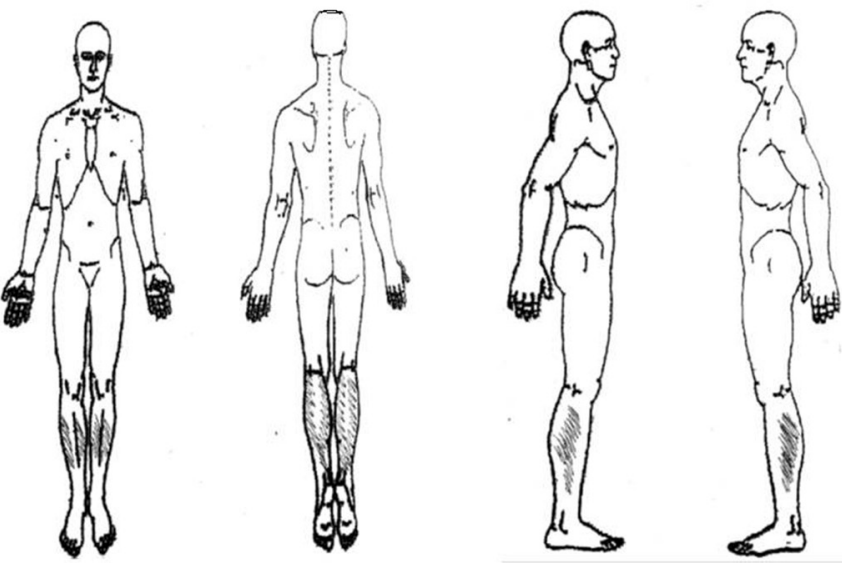

Figure 4 An illustration of a patient pain drawing on a human outline made by a patient with exercise-induced leg pain.

of comorbidity. Most patients marked multiple pain locations on the leg (figure 4). In the present study, we found that among 168 patients with CACS, 89 patients $(53 \%)$ had one or more additional diagnoses. This result is high but in line with previous studies that have reported comorbidity of $42 \%{ }^{6}$ and $47 \% .^{7}$ Undiagnosed and untreated comorbidity is a possible cause of unsatisfactory outcome after fasciotomy. If the patient is only operated on with fasciotomy for CACS, the codiagnoses are left untreated. PPD is most helpful to identify the number of pain locations and shows a clear picture whether it comes to one or several areas of pain. This could facilitate in the preoperative planning when it is obvious that the patient is suffering from one or more additional diagnosis/ diagnoses. The patient could also realise the presence of comorbidity while studying the drawing together with the physician. Altogether, comorbidity may play a significant role in the diagnosis, treatment and results for surgical outcome. In our experience, PPD is a valuable clinical instrument that facilitates the process of reaching a correct diagnosis of exercise-induced leg pain. Another factor that makes the diagnostic process difficult is that the patients are frequently free from symptoms at rest during the consultation.

Detailed anatomical knowledge is important when it comes to understanding the cause of leg pain. Twenty-nine per cent of the patients with CACS had MTS. These patients indicated pain over the anterior part of the leg and over the medially on the midportion of the tibia. MTS is clinically seen as pain over the middle third of the posteromedial portion of the tibia that is tender at palpation. In $11 \%$ of the patients with CACS, PTS was diagnosed. The patients indicated pain on the distal third of the leg, laterally. This finding agrees with a previous report. ${ }^{6}$ Diagnoses such as muscle strains and partial muscle rupture, popliteal entrapment, venous insufficiency and suspected stress fractures were found in $13 \%$ of the patients with CACS.

\section{Limitations}

This study only considered the organic genesis of pain. No evaluation was added when it came to whether the drawings should be interpreted with regard to psychological aspects. Apart from exercise-induced leg pain, the patients were healthy individuals involved in full working activity. The procedure for diagnosing CACS among the causes of exercise-induced leg pain requires further development. The present PPD form can most probably be improved in order to increase the likelihood of correctly establishing a more detailed diagnosis in patients with exercise-induced leg pain.

\section{CONCLUSION}

PPD might be an additional valuable instrument in diagnosing the causes of exercise-induced leg pain. It is useful in identifying CACS with and without comorbidity.

Contributors KR is the principal investigator in charge of the project. QZ, JK and JS have all been involved in designing the intervention and the evaluation. KR prepared the initial draft of the manuscript, and all other authors have contributed. All authors have critically reviewed and approved the final version of the manuscript.

Competing interests None declared.

Ethics approval The study protocol was approved by Research Ethics Committees at the University of Gothenburg, ID no. 617-08.

Provenance and peer review Not commissioned; externally peer reviewed.

Data sharing statement Data are available from the corresponding author on reasonable request.

Open Access This is an Open Access article distributed in accordance with the Creative Commons Attribution Non Commercial (CC BY-NC 4.0) license, which permits others to distribute, remix, adapt, build upon this work non-commercially, and license their derivative works on different terms, provided the original work is properly cited and the use is non-commercial. See: http://creativecommons.org/ licenses/by-nc/4.0/

(C) Article author(s) (or their employer(s) unless otherwise stated in the text of the article) 2018. All rights reserved. No commercial use is permitted unless otherwise expressly granted.

\section{REFERENCES}

1. Palmer H. Pain charts; a description of a technique whereby functional pain may be diagnosed from organic pain. $N Z M e d J$ 1949;48:187-213.

2. Hägg $O$, Fritzell $P$, Hedlund $R$, et al. Pain-drawing does not predict the outcome of fusion surgery for chronic low-back pain: a report from the Swedish Lumbar Spine Study. Eur Spine J 2003;12:2-11.

3. Detmer DE, Sharpe K, Sufit RL, et al. Chronic compartment syndrome: diagnosis, management, and outcomes. Am J Sports Med 1985;13:162-70.

4. Allen MJ, Barnes MR. Exercise pain in the lower leg. Chronic compartment syndrome and medial tibial syndrome. J Bone Joint Surg Br 1986;68:818-23.

5. Barnes M. Diagnosis and management of chronic compartment syndromes: a review of the literature. Br J Sports Med 1997;31:21-7.

6. Styf J. Diagnosis of exercise-induced pain in the anterior aspect of the lower leg. Am J Sports Med 1988;16:165-9.

7. Zhang Q, Rennerfelt K, Styf J. The magnitude of intramuscular deoxygenation during exercise is an unreliable measure to diagnose the cause of leg pain. Scand J Med Sci Sports 2012;22:690-4.

8. Clanton TO, Solcher BW. Chronic leg pain in the athlete. Clin Sports Med 1994;13:743-59.

9. Edwards PH, Wright ML, Hartman JF. A practical approach for the differential diagnosis of chronic leg pain in the athlete. Am J Sports Med 2005;33:1241-9.

10. Tucker AK. Chronic exertional compartment syndrome of the leg. Curr Rev Musculoskelet Med 2010;3:32-7.

11. Hutchinson MR, Ireland ML. Common compartment syndromes in athletes. Sports Medicine 1994;17:200-8. 
12. Styf JR, Körner LM. Diagnosis of chronic anterior compartment syndrome in the lower leg. Acta Orthop Scand 1987;58:139-44.

13. Styf $J$, Morberg P. The superficial peroneal tunnel syndrome. Results of treatment by decompression. J Bone Joint Surg $\mathrm{Br}$ 1997;79:801-3.

14. Wallensten R, Eriksson E. Intramuscular pressures in exerciseinduced lower leg pain. Int J Sports Med 1984;5:31-5.

15. Detmer DE. Chronic shin splints. Sports Medicine 1986;3:436-46.

16. Pedowitz RA, Hargens AR, Mubarak SJ, et al. Modified criteria for the objective diagnosis of chronic compartment syndrome of the leg. Am J Sports Med 1990;18:35-40.

17. Yates $\mathrm{B}$, White $\mathrm{S}$. The incidence and risk factors in the development of medial tibial stress syndrome among naval recruits. Am J Sports Med 2004;32:772-80.
18. Styf JR, Körner LM. Microcapillary infusion technique for measurement of intramuscular pressure during exercise. Clin Orthop Relat Res 1986;207:253-62.

19. Zhang Q, Jonasson C, Styf J. Simultaneous intramuscular pressure and surface electromyography measurement in diagnosing the chronic compartment syndrome. Scand J Med Sci Sports 2011;21:190-5.

20. Gershuni DH, Yaru NC, Hargens AR, et al. Ankle and knee position as a factor modifying intracompartmental pressure in the human leg. J Bone Joint Surg Am 1984;66:1415-20.

21. Reigo T, Tropp H, Timpka T. Pain drawing evaluation--the problem with the clinically biased surgeon. Intra- and interobserver agreement in 50 cases related to clinical bias. Acta Orthop Scand 1998;69:408-11. 